\title{
Higher performance and lower complexity turbo decoding scheme for 4G-LTE using unpunctured turbo trellis-coded modulation
}

\author{
ELarbi Abderraouf, Abdesselam Bassou, Mohamed Rida Lahcene \\ Department of Electrical Engineering, Tahri Mohammed University-Bechar, Algeria
}

\section{Article Info}

Article history:

Received Aug 4, 2019

Revised Oct 6, 2019

Accepted Oct 20, 2019

\section{Keywords:}

4G-LTE

Decodingcomplexity

Turco-code

UTTCM

\begin{abstract}
Mobile communications have become necessities and even everyday commodities. In the previous decades they evolved from a technology reserved for a limited number of individuals to the current systems that are everywhere in our lives. The task of developing mobile technologies has also evolved from a national or regional focus to a complex and growing mission, supported by global standards development organizations such as 3GPP (3rd Group Partnership Project). Thanks to the success of smart phones and mobile-ready laptops, data traffic has recently grown exponentially, and the demand for mobile data has risen very dramatically. These requests in large capacity can only be satisfied by a high efficiency and a very good optimization of the infrastructures of the mobile networks, while taking into account the constraints which are the power, bandwidth and a limited complexity. Through this research, we present everything related to the simulation of the $4 \mathrm{G}$ mobile network system (LTE), which can provide high data flow with good quality, through three model channels known as (EPA, EVA, ETU). In this work we focus on the block 'iterative decoding channel encoder' in the LTE system, where the iterative channel coding called 'Turbo-code' (TC) is substituted by the iterative coding channel called 'Unpunctured Turbo Trellis-coded Modulation' (UTTCM). The simulation results showed that with less decoding complexities, UTTCM's LTE system gives good performance (in terms of BER).
\end{abstract}

Copyright $@ 2020$ Institute of Advanced Engineering and Science. All rights reserved.

\section{Corresponding Author:}

Elarbi Abderraouf,

Department of Electrical Engineering,

Tahri Mohammed University-Bechar, Algeria.

Email: elarbiabderraouf12@gmail.com

\section{INTRODUCTION}

From Morse communications in the middle of the 19th century to the current mobile telephony, to Shannon's work [1], interest in communication systems has grown steadily. However, even though the ability to communicate wirelessly using Hertzian waves was discovered more than a century ago, the phenomenon accelerated in the late 20th century, with the democratization of digital systems and the deployment of mass-market cellular telephony. However, faced with the current convergence, it is at the level of all-in-one devices or different digital streams (voice, images, video, messages and data) grouped on the same transmission protocol as IP (Internet Protocol), the multiplicity of systems and standards can harm the attractiveness of recent developments. Refocusing on the needs of the user and the transparency of the accesses, while making the minimum compromise on the quality and the cost per bit transmitted, becomes essential to ensure a craze towards the so-called 4th generation $(4 \mathrm{G})$ systems, which will have to provide mobile broadband with associated services in a suitable way. 
Researchers around the world have investigated the efficiency of LTE system using simulation. In [2], the author has implemented the physical layer using Matlab Simulink in order to emphasize high spectral efficiency, high peak data rates, low latency, and frequency flexibility. Considering that, LTE schedulers should be able to meet the needs of machine-to-machine (M2M) devices such as time constraints and specific Quality of Service (QoS) requirements, the authors of [3] have proposed an LTE scheduler that proved, through simulation, that it is able to reach the lowest Packet Loss Rate (PLR) and deliver highest throughput.

To address the constraints with limited bands and powers, Orthogonal Frequency Division Multiplex (OFDM) multi-carrier algorithms are among the main candidates for the spectral efficiency race. Their combination is particularly suitable, and allows considering a rise in data flow rate while maintaining a reasonable reception complexity. Indeed, multi-carrier techniques [4] make it possible to ideally use the radio resource and to comply with strict spectral constraints by distributing the power appropriately. Moreover, combined with coding, they ensure robustness by exploiting time-frequency diversity. The progress of coding-channel techniques, in particular with the appearance of the turbo principles in the article by Berrou [5], makes it possible to approach the theoretical limits provided by Shannon.

Therefore, any approach to the design and optimization of communication systems must not only lead to a compromise between spectral efficiency, but also to the resistance of these systems against transmission errors. The most important factors that lead to the quality and durability of the mobile cellular network (LTE) are related to the power and efficiency of the block (channel coding) on the transmission chain. Advanced research in the area of error correction codes has confirmed that the most powerful and efficient codes that have a better decoding algorithm (in terms of corrective capability) and the least complexities, are the ones that are limited to codes that use the iterative calculation algorithm. An optimal code can have a great capacity of the correction of errors, if its complexity is weak [6], as we can reduce the decoding complexity while preserving the same performances of this code as those described in [7-9]. Hence the practical advantage of localized iterative decoding in that powerful codes can now be decoded with reasonable complexity, so much of the research is focused on evaluating iterative decoding performance for the future LTE network systems. Proakis considered in [10] that turbo-code is an evolution of the convolutional encoding technology used in all previous standards with impressive performance in terms of capacity in nearby channels. Since the first publication on the turbo codes, various works have been done. Most of these works illustrated their performance or presented optimization methods using binary modulations. However, the concern to transmit at high rates while being confined to a finite bandwidth has led some authors [11-13] to consider the application of turbo codes for modulations with high spectral efficiency.

An attempt to improve the performance in sense of Bit Error Rate (BER) of LTE decoder has been proposed in [14]. The authors have proposed an architecture of reversed-trellis tail-biting convolutional codes [15] (RT-TBCC) for LTE communication standardthatdemonstrates promising results in hardware implementation and performance. This solution is a significant decrease in complexity compared to fixed-tail maximum-likelihood (FTML) decoder.

The main goal of this work is to improve the performance of the $4 \mathrm{G}$ network using a new iterative coding technology, which is efficient and less complex, called UTTCM [16] instead of turbo-code. UTTCM is among iterative codes with high spectral efficiency and which contains two TCM encoders linked together in parallel. Trellis-coded modulations (TCM) was invented by Ungerboeck in 1982 [17] and UTTCM codes was the result of a continuous series of innovations from the TTCM as described in $[18,19]$ to the PTTCM as described in [20-22]. UTTCM codes are very effective for communications on limited band channels [23].

\section{STRUCTURE OF THE LTE SYSTEM}

The physical layer of LTE technology, depicted in Figure 1, is an efficient way of sending data and control signals between base station and the mobile user. LTE uses many advanced technologies, including Orthogonal Frequency Division Multiplexing (OFDM). Besides these on the uplink, it uses single carrier Frequency Division Multiple Access (SC-FDMA), while on the downlink, it uses Orthogonal Frequency Division Multiple Access (OFDMA). In this section we will model the different blocks of the physical layer in the case of a downlink (link between an eNodeB and a UE) through the downstream transport channels $(\mathrm{PDSCH})$. For this, we will consider the three entities involved: the transmitter, the propagation channel and the receiver.

In the transmitter side, the data coming from the upper layers are encapsulated in the form of frames [24-26] called "transport block" before their passage in the radio link and their transmission whose duration is characterized by the Transmission Time Interval (TTI). The Transport Block Size or TBS depends on the number of physical resource blocks (PRBs) and the modulation and coding scheme adopted. The transmitter 
starts with resource data that are grouped as transport blocks. In each TTI, a transport block will be transferred first to the coding portion of the channel that consists of two encoders CRC and a Turbo encoder. knowing that the most used CRC calculations are designed to always be able to detect errors of certain types, such as those due for example to interference during transmission, CRC is used at the beginning of channel coding [27]. Next, the rate adaptation block combined with the hybrid ARQ retransmission technique is a kind of coordinator between the channel coding and the downlink shared physical channel blocks [28]. Then the code word transmitted to the physical channel in a subframemustbe scrambled before the modulation to cope with the interference by performing the XOR operation of the input bits with a scrambling code [29]. At the end of the transmitter chain, we use the so-called modulation that allows bits to be associated with modulation symbols, such that each code word must be modulated with one of the modulations (QPSK, 16QAM, 64QAM). Then we send the result of modulation via the OFDM technique [30] through radio channels to reach the receiver that does the reverse processing.

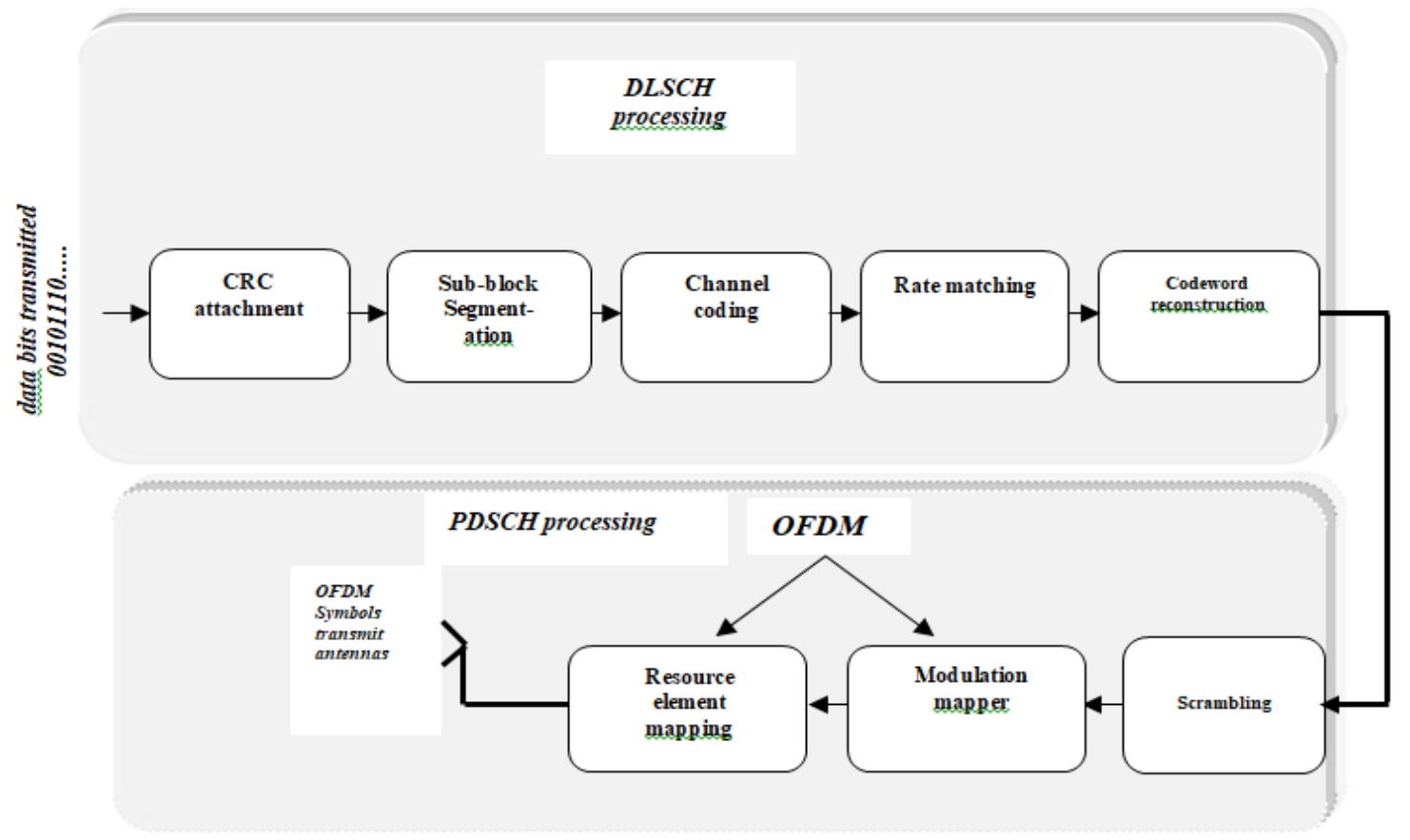

Figure 1. Physical layer specifications in LTE

\section{CODING OF THE CLASSICAL CHANNEL IN LTE}

Radio propagation channel models are considered to be a more important factor in the simulation of radio transmission systems. Therefore, all standards for design and optimization recommend channel models for specific propagation environments. Knowing that these channel models are characterized by path loss mitigation, shadow fading, and multipath fading, our interest in this work is centered on the conditions of propagation of multipath fading present in a typical LTE channels, and their impact on the estimation of the temporal delay of the signals. From the definition of Tapped-Delay Line (TDL) models, 3GPP standards have determined the impulse response of the LTE channel by the following formula:

$$
h(\tau, t)=\sum_{k=1}^{K} a_{k} \delta\left(\tau, \tau_{k}\right)
$$

while each path is characterized by a constant delay, a relative power and also by a Doppler spectrum, where $k$ is the number of the path and $\tau_{k}$ is the path delay $k$ relative to the first path and $a_{k}$ is the complex amplitude distributed by Rayleigh for each path, which follows a conventional Jakes Doppler spectrum $S(f)$ :

$$
S(f) \propto \sqrt{\frac{1}{1-\left(f^{f} / f_{D}\right)^{2}}} \text { and } f \in\left[-f_{D}, f_{D}\right]
$$


The 3GPP standard in [31], was limited to the use of three models of the main channels, represented in Pedestrian A and Vehicular A channels in [32], and the Typical Urban channel (TU) in [33], which correspond exactly and respectively to the environments characterized by a low, medium and large delay difference, knowing that these channels exploits a bandwidth that reaches $5 \mathrm{MHz}$ or higher [34]. As described in TS 36.101 [35] and TS 36.104 [36] and by means of the 3GPP and ITU standard, the LTE system is adopted on three models of propagation channels essential for simulation and testing. These models are defined as follow: the Extended Pedestrian A (EPA), Extended Vehicular A (EVA) and Extended Typical Urban (ETU) channel models. The following Table 1 shows the maximum Doppler shifts for each model representing low, medium, and high mobile conditions.

Table 1. LTE Tapped-delay Line Channel Models Parameters

\begin{tabular}{ccccccc}
\hline Tap no. & \multicolumn{2}{c}{ EPA channel } & \multicolumn{2}{c}{ EVA channel } & \multicolumn{2}{c}{ ETU channel } \\
& $\tau(\mathrm{ns})$ & SMR $(\mathrm{dB})$ & $\tau(\mathrm{ns})$ & SMR $(\mathrm{dB})$ & $\tau(\mathrm{ns})$ & SMR $(\mathrm{dB})$ \\
\hline 1 & 0 & 0.0 & 0 & 0.0 & 0 & -1.0 \\
2 & 30 & -1.0 & 30 & -1.5 & 50 & -1.0 \\
3 & 70 & -2.0 & 150 & -1.4 & 120 & -1.0 \\
4 & 90 & -3.0 & 310 & -3.6 & 200 & 0.0 \\
5 & 110 & -8.0 & 370 & -0.6 & 230 & 0.0 \\
6 & 190 & -17.2 & 710 & -9.1 & 500 & 0.0 \\
7 & 410 & -20.8 & 1090 & -7.0 & 1600 & -3.0 \\
8 & & & 1730 & -12.0 & 2300 & -5.0 \\
9 & & & 2510 & -16.9 & 5000 & -7.0 \\
\hline
\end{tabular}

\section{SIMULATION RESULTS ON THE LTE SYSTEM}

The objective of this part is to simulate the physical layer of the mobile network of the 4th generation (LTE) downlink with Matlab tools by varying the different parameters, which will allow us to highlight the contribution of this new performance technology such as data flow rate, Bit Error Rate (BER), etc. We focus (in the transmission chain shown in Figure 1) on the channel coding block to improve the performance quality of the LTE system, firstly by using the turbo-coder as an error-correcting code.

\subsection{The used Parameters}

The Table 2 as shown in parameters used during the simulation are given as follow:

Table 2. Parameters used in the Simulation

\begin{tabular}{cc}
\hline Parameter & Value \\
\hline size of frame transmitted & 3869 \\
FFT size & 1024 \\
No of resource blocks & 50 \\
No of Transmit and received antennas & $1 \times 1$ \\
Cell ID & 17 \\
Cyclicprefix & Normal \\
Duplex mode & TDD(Time Division Duplex) \\
Downlinkchannel & PDSCH (Physical downlink shared channel) \\
Channel bandwidth & 10 MHz \\
Channel model & EVA / EPA / ETU \\
Doppler shift & f(EVA) $=222.22 / \mathbf{f}($ EPA $)=5.55 / \mathbf{f}($ ETU $)=5.55$ \\
Modulation & QPSK, 16QAM, 64QAM \\
Equalization mode & Zero-forcing \\
\hline
\end{tabular}

From the simulation results, the bit error rate of a digital communication system is an important parameter used to quantify the integrity of the data transmitted by the system. Figures 2,3 and 4 show the performance of the LTE system using different modulations: QPSK, 16-QAM and 64-QAM over three channels EPA, EVA, ETU using turbo coded as channel coding where its coding rate equals to 1/3. The decoding is done using the Log-MAP algorithm with a number of decoding iterations equals to 5.

From Figures 2, 3 and 4, it can be deduced that, on all the channels of the LTE system simulation (EPA, EVA, ETU), the performance of the system decreases when the constellation size and the spectral efficiency increase. 


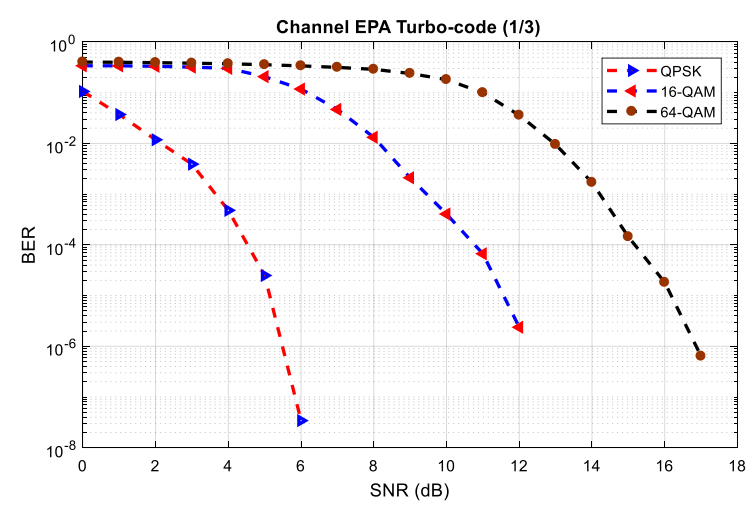

Figure 2. LTE system performance for different modulations, using the standard turbo-code channel coding for 5 decoding iterations on EPA channel

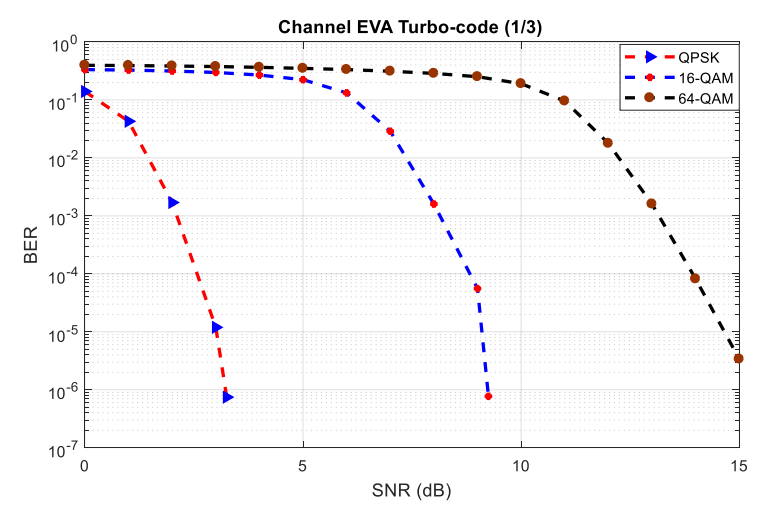

Figure 3. LTE system performance for different modulations, using standard turbo-code channel coding for the 5th iteration of EVA channel decoding

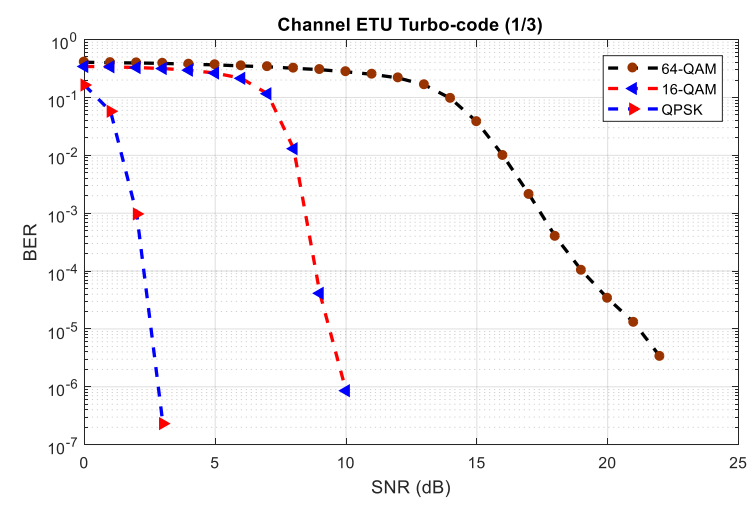

Figure 4. LTE system performance for different modulations, using standard turbo-code channel coding for the 5th iteration of ETU channel decoding

\subsection{UTTCM Encoder Performance on the LTE System}

The performance of a coded modulation scheme for a non-frequency selective Rayleigh channel is strongly dependent on the minimum Hamming distance between symbols of the transmitted code. In [37], Al-Semari and Fuja proposed I-Q TCM (Modulations in Phase Trellis and Quadrature Coded Modulation) schemes that have far greater symbol-to-symbol Hamming distances than conventional Trellis coded modulation schemes. They showed that large gains could be obtained by using simple convolutional codes optimized in terms of minimum Hamming distance in combination with coding and mapping on I and Q channels. The TCM coder was chosen by maximizing the free Euclidean distance $\left(d_{f}\right), d_{f}$ is calculated by an algorithm that searches, over the TCM trellis, the path that maximizes $d_{f}$. In an IQ turbo-encoder, each group of information bits is associated with its coded symbols which are obtained alternately either at the output of the first turbo-encoder, or at the output of the second. The bits coded at the output of the two coders are modulated separately on the in-phase and quadrature channels of the desired constellation following a Gray mapping. In this part, we introduce the UTTCM coding structure, which is used during downlink transmission of the LTE system (based on TCM codes).

The UTTCM encoder is a parallel concatenation of two TCM coders of efficiency $m /(m+1)$ separated by a symbol interleaver [16]. At the input of the mapper, we find $m$ systematic bits, a bit of parity from the external encoder and an unknown deinterlaced bit from the internal encoder successively. This leads to the use of a constellation $2^{m+2}$ point (for simulations, M-QAM modulation is adopted) [16]. The UTTCM encoder works, in this case, with a spectral efficiency of $m b p s$.

Figure 5 illustrates the structure of the LTE system when using the UTTCM encoder. The difference between the two structures of the LTE system that use the encoders (turbo-code and UTTCM) is the absence of the mapping scheme in the LTE transmission chain. 


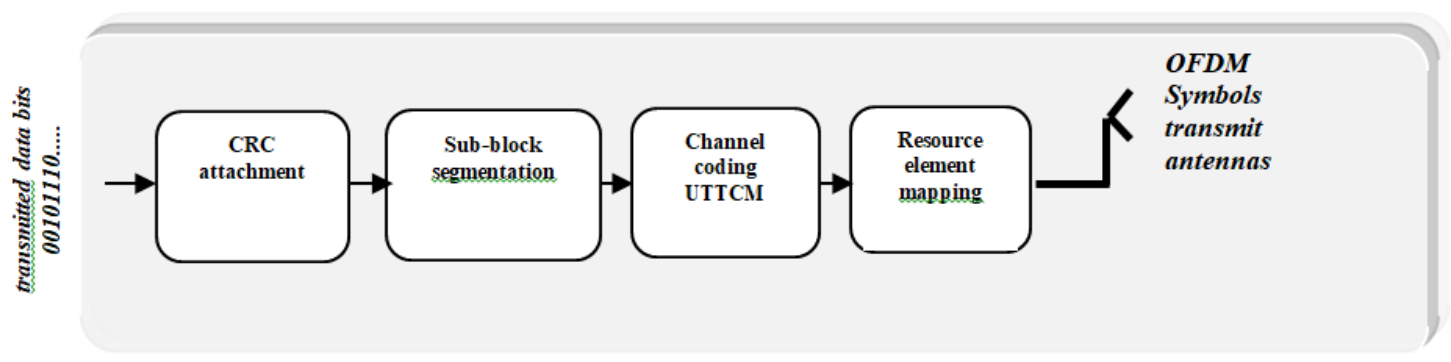

Figure 5. Physical layer specifications in LTE using UTTCM

The Table 3 shows the decoding complexity of the 8-state UTTCM encoder and the standard 8-state turbo-code encoder (coding rate $r=1 / 3$ ) used in the previous simulation, for a spectral efficiency of $2 \mathrm{bps}$. It is clearly shown from Table 3 that the substitution decoding scheme (UTTCM) is less complex than Turbocode.

Table 3.Turbo-code and UTTCM Comparison as a Function of Decoding Complexity for

\begin{tabular}{cccc}
\multicolumn{4}{c}{ 16-QAM and 64-QAM } \\
\hline \multirow{4}{*}{ Channel encoder } & Turbo-code & UTTCM \\
& Number of states & \multicolumn{2}{c}{8 states } \\
& Modulation & 64QAM & 16QAM \\
\hline \multirow{3}{*}{ operations } & Addition & 248352 & 98120 \\
& Subtraction & 410624 & 105704 \\
& Multiplication & 497958 & 122574 \\
& comparison & 941444 & 233540 \\
\hline
\end{tabular}

In this section we present and interpret the simulation results of UTTCM coding techniqueapplied on the LTE system transmission chain for the three fading channels (EPA, EVA, ETU) and for the 16QAM modulation shown in Figure 6 with two types of mappings (Ungerboeck-Gray and Gray). We will show that the UTTCM encoding technique is very efficient in terms of BER compared to the standard Turbo-code channel coding when we keep the same spectral efficiency between the two coding techniques.

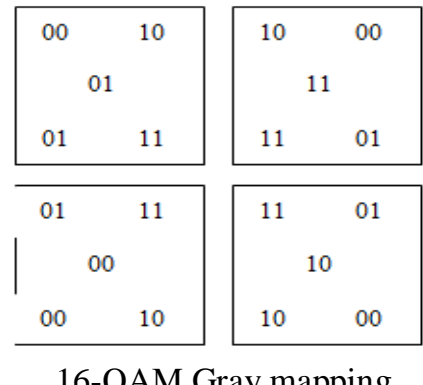

16-QAM Gray mapping

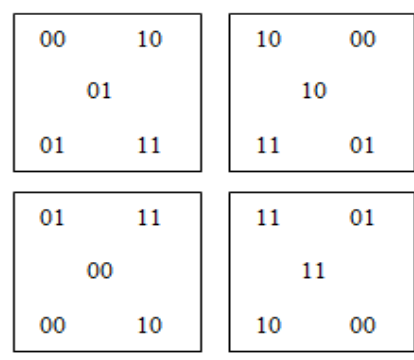

16-QAM Ungerboeck-Gray

Figure 6. Constellations of 16-QAM with Gray mapping and Ungerboeck-Gray mapping

According to the results of simulations shown in Figure 7, it can be seen over the three fading channels (EPA, EVA and ETU) that, for the same spectral efficiency of $2 \mathrm{bps}$, the UTTCM coder has a good performance compared to the turbo-code coder during downlink transmission of LTE system despite the very low complexity of UTTCM encoder decoding relatively to turbo-code decoder.

The existing encoding gain between the UTTCM encoder and Turbo-code curves has been measured when BER $=10^{-5}$. For the EPA channel we have benefited from $\mathrm{G} 1=4.71 \mathrm{~dB}$, for the EVA G2 = $4.56 \mathrm{~dB}$ and for the ETU G3 $=7.54 \mathrm{~dB}$, so the LTE system with UTTCM channel encoding has better performance than the LTE system with standard Turbo-code encoding on all simulation channels (EPA, EVA, ETU). 


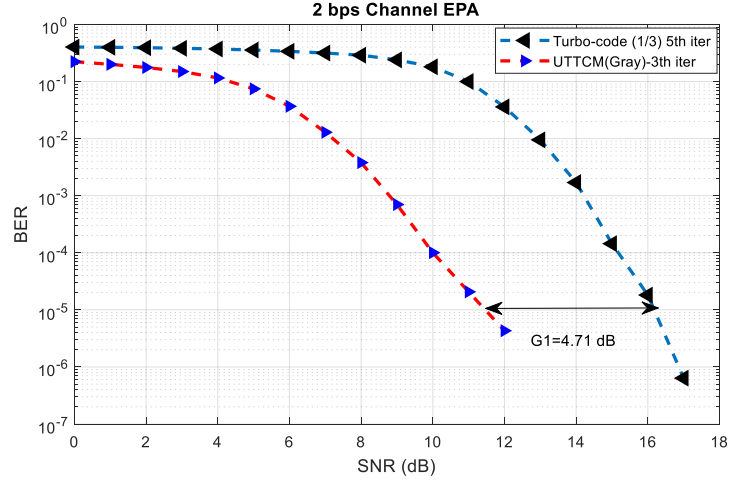

(a)

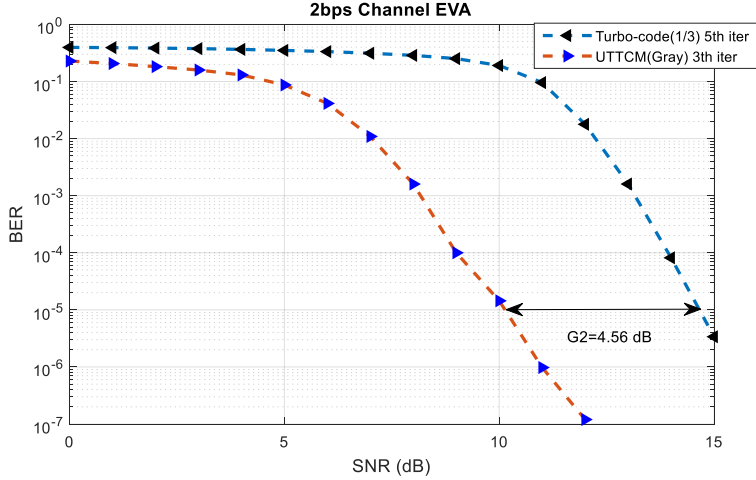

(b)

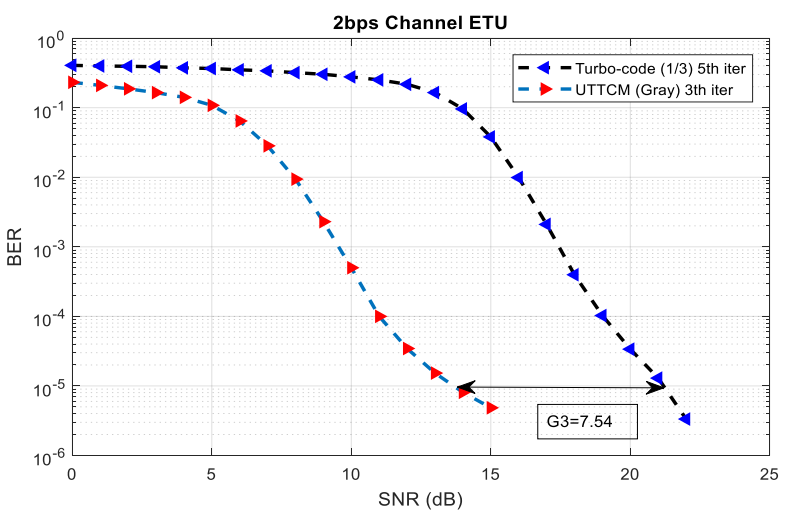

(c)

Figure 7. Performance comparison between the LTE system using the modulation UTTCM encoder (16QAM) for the 3rd decoding iterations and the LTE using the standard Turbo-code for the 5th iteration (64QAM) on: (a) the EPA channel, (b) the EVA channel, (c) the ETU channel

\subsection{Influence of Iteration Number with Decoder Complexity on the EPA Channel}

Figure 8 shows the performance comparison between the results obtained from the UTTCM encoder and the standard Turbo-code encoder, as well as the influence of the number of iterations, for the EPA channel.

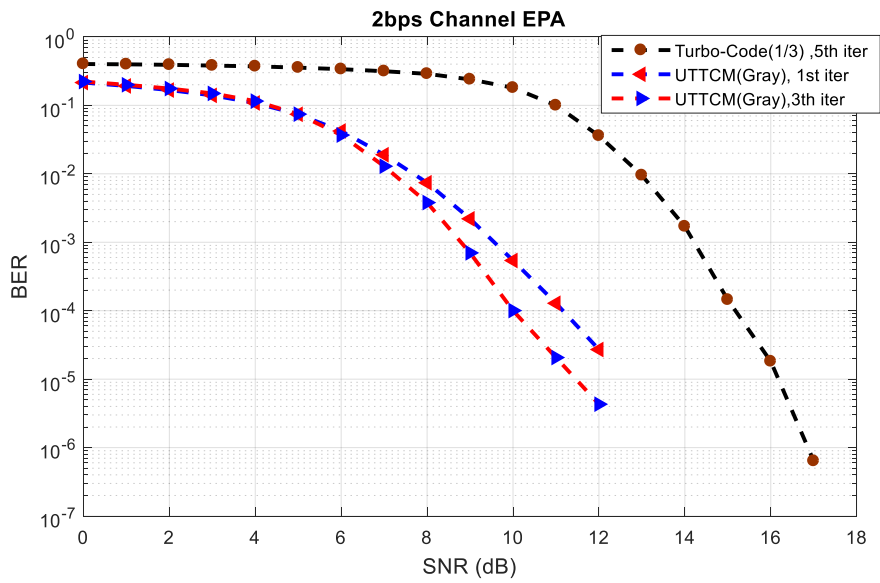

Figure 8. LTE system performance comparison using the UTTCM encoder for 1 and 3 decoding iterations and the LTE using the Turbo-code encoder for the 5th iterations on the EPA channel 
This result indicates that the UTTCM encoder gave higher performance compared to the turbocoder. Despite the fact that the degree of complexity of the UTTCM decoder is much less than the turbo-code decoder, this result also shows that the more iterations, the higher the performance, however the increase of numbers of iterations lead to the increase of the degree of complexity, and hence the cost of decoding. Therefore, the relationship between the complexity rate and the degree of cost is proportional.

\subsection{Effect of the Constellation Mapping on the Performance}

To make signal sequences more resistant to noise, they must be very different from one to another. In other words, the Euclidean distance between the different signals taken in their space must be as big as possible. Figure 9 shows that the constellation that uses Gray mapping gives better performance (in terms of BER) than the constellation that uses Ungerboeck-Gray mapping over all simulation channels.

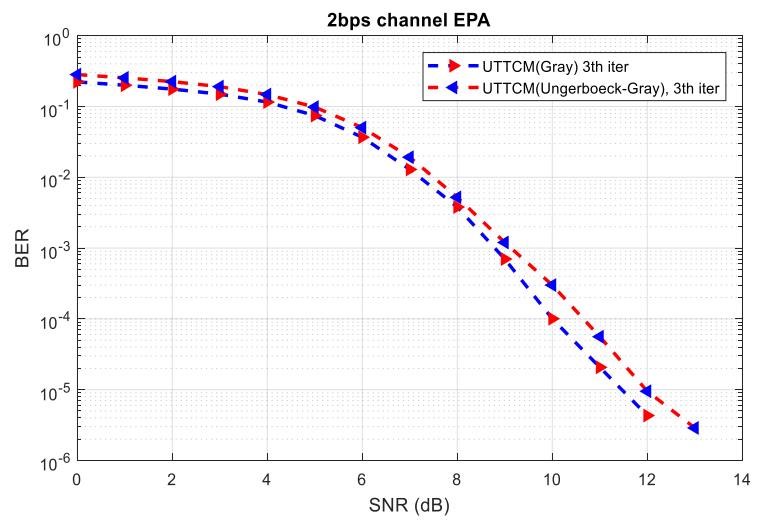

(a)

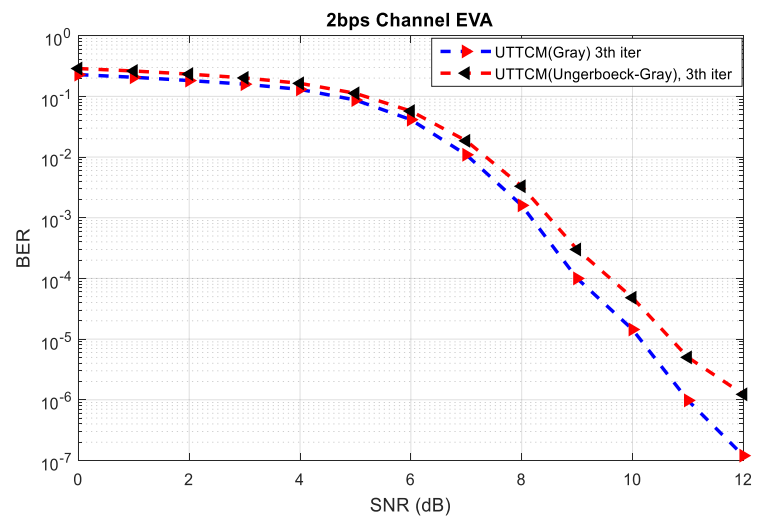

(b)

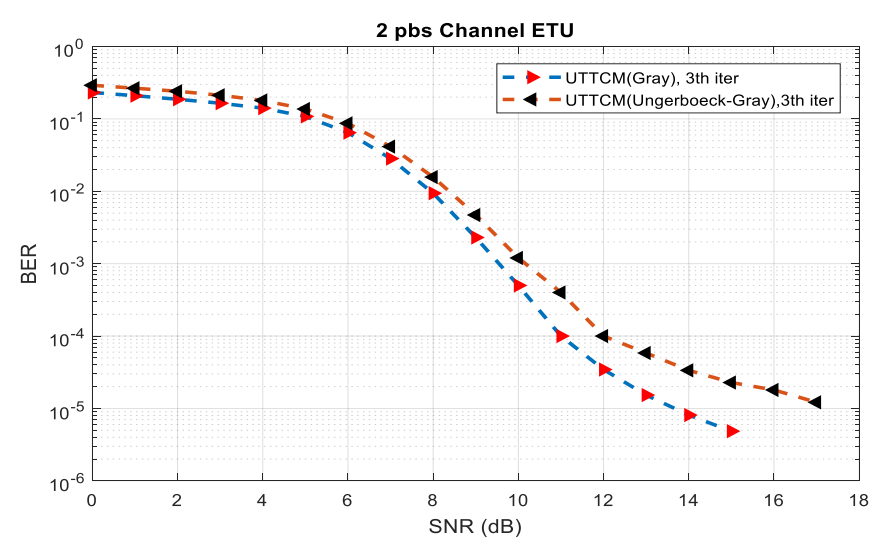

(c)

Figure 9. Performance comparison between gray and Ungerboeck-Gray constellation of the UTTCM coder used in the downlink of the LTE system on the channel: (a) EPA, (b) EVA, (c) ETU

\section{CONCLUSION}

At the physical layer level, the LTE system makes it possible to exploit the propagation channel with great flexibility. The flexibility of the configuration offers many advantages (scalability according to the mobile receiver's capabilities, adaptation to the environment, etc.). The potential of LTE system techniques have been evaluated in order to satisfy the constraints of spectral efficiency, robustness or even compatibility with simple and economical receivers, in various environments. This framework realized in this article focuses mainly on analysis and simulation for optimizing and improving the performance of LTE systems against transmission errors in model channels (EPA, EVA, and ETU). The results of the simulations have proved that the UTTCM encoding technique brings a significant gain in terms of performance quality and the degree of complexity i.e. complexity, relative to the turbo-code. 


\section{ACKNOWLEDGEMENTS}

We want to thank very much the members and the directors of LTIT Laboratory of University of Bechar for their great availability, their encouragements, and their precious advice. I would also like to thank the editor and anonymous reviewers for their comments and suggestions.

\section{REFERENCES}

[1] C. E. Shannon, "A mathematical theory of communications," Bell system technical journal, Jul. 1948, pp. $379-423$.

[2] F. F Al Azzawi, "LTE RF receiver modeling and each part testing with MATLAB Simulink," Indonesian Journal of Electrical Engineering and Computer Science (IJEECS), vol. 14(3), June 2019, pp.1251-1257.

[3] M. Ouaissa and A. Rhattoy, "QoS hybrid uplink scheduler based on service type for M2M communications in LTE networks," Indonesian Journal of Electrical Engineering and Computer Science (IJEECS), vol. 14(3), June 2019, pp. 1460-1470.

[4] R. W. Chang, "synthesis of band-limited orthogonal signal for multichannel data transmission," Bell system technical Journal, vol. 45, Dec. 1966, pp. 1775-1796.

[5] C. Berrou, A. Glavieuxand P. Thitimajshima, "Near Shannon limit error-correcting coding and decoding: Turbocodes," In EEE International conference on communication, May 1993, pp. 1064-1070.

[6] N. Chen and Z. Yan, "Complexity analysis of Reed-Solomon decoding over GF $\left(2^{m}\right)$ without using syndromes," EURASIP Journal on Wireless Communications and Networking, vol. 2008, pp. 1-11.

[7] J. Bellorado and A. Kavcic, "Low complexity decoding algorithms for Reed-Solomon codes-Part I: An algebraic soft-in-hard-out chase decoder." IEEE Transaction on Information Theory, vol. 56(3), Mar. 2010, pp. 945-959.

[8] J. Bellorado, A. Kavcic, M. Marrow and L Ping, "Low complexity soft-decoding algorithms for Reed-Solomon codes-Part II: Soft-Input Soft-Output iterative decoding," IEEE Transaction on Information Theory, vol. 56(3), Mar. 2010, pp. 960-967.

[9] D. Matolak and S. Wilson, "Variable-complexity trellis decoding of binary convolutional codes," IEEE Transaction on Communication, vol. 44(2), Feb. 1996, pp. 121-126.

[10] J. G. Proakis, "Digital Communications”, Ed. Mc. Graw-Hill, New York, 2001.

[11] S. Le Goff, A. Glavieux and C. Berrou, "Turbo-codes and high spectral efficiency modulation," in Proc. IEEE International Conference on Communication, May 1994, pp. 645-649.

[12] S. Benedetto, D. Divsalar, G. Montorsi and F. Pollara, "Parallel concatenated trellis coded modulation,"in Proc. IEEE International Conference on Communication, May 1996, pp. 974-978.

[13] P. Robertson and T. Wörz, "Coded modulation scheme employing turbo codes,"Electronics Letters, vol. 31, Aug. 1995, pp.1546-1547.

[14] T. Adiono, A. Z. Ramdani and R. V. W. Putra, "Reversed-trellis tail-biting convolutional code (RT-TBCC) decoder architecture design for LTE," International Journal of Electrical and computer Engineering (IJECE), vol. 8(1), Feb. 2018, pp. 198-209.

[15] A. Z. Ramdani and T. Adiono, "A novel algorithm of tail biting convolutional code decoder for low cost hardware implementation", in Proc. International Symposium on Intelligent Signal Processing and Communication Systems (ISPACS), November 2015, pp. 241-245.

[16] A. Bassou, A. Djebbari and M. Benaissa, "Design of unpunctured turbo trellis-coded modulation," AE $\ddot{U}$ International Journal of Electronics and Communications, vol. 67(3), Mar. 2013, pp. 223-232.

[17] G. Ungerboeck, "Channel coding with multilevel/phase signaling," IEEE Transaction on Information Theory, vol. 25(1), Jan. 1982, pp. 55-67.

[18] P. Robertson and T. Wörz, "Coded modulation scheme employing turbo codes," Electronics Letters, vol. 31(2), Aug. 1995, pp. 1546-1547.

[19] P. Robertson and T. Woerz, "Bandwidth-efficient turbo trellis coded modulation using punctured component codes," IEEE J. on Selected Areas in Communication, vol. 16(2), Feb. 1998, pp. 206-218.

[20] D. Divsalar and F. Pollara, "On the Design of Turbo Codes," JPL TDA Progress Report 42-123, Nov. 1995, pp. 99-121.

[21] S. Benedetto, D. Divsalar, G. Montorsi and F. Pollara, "Bandwidth efficient parallel concatenated coding schemes," Electronics Letters, vol. 31(24), Nov. 1995, pp. 2067-2069.

[22] S. Benedetto, D. Divsalar, G. Montorsi and F. Pollara, "Parallel concatenated trellis codes modulation," International Conference on Communications, Dallas, USA, June 1996, pp. 974-978.

[23] M. R. Lahcene, A. Bassou, M. Beladgham and A. Taleb-Ahmed, "On the employment of unpuncturedturbo-trellis coded modulation channel coding to improve the performance of WiMaxsystem,"International Journal on Communications Antenna and Propagation (IJCAP), vol. 8(3), June 2018, pp. 248-256.

[24] Y. Bouguen, É. Hardouin and W. François-Xavier, LTE et les réseaux 4G, Ed. Eyrolles,Paris, 2012.

[25] Larmo, A. Lindstrom, M. Meyer, M. Pelletier, G. Torsner and J. Wiemann, "The LTE link-layer design", IEEE Communications Magazine, vol. 47(4), Apr. 2009, pp. 52-59.

[26] F. Khan, "LTE for 4G Mobile Broadband", Cambridge University Press, 2009.

[27] 3GPP TS 36.212, "Evolved Universal Terrestrial Radio Access (E-UTRA); Multiplexing and channel coding," V9.2.0, May 2010.

[28] X. GUO and P. SONG, "Simulink based LTE system simulator," Rapport N. EX097/2010.

[29] 3GPP TS 36.211, "Evolved universal terrestrial radio access (E-UTRA); physical channels and modulation," V9.2.1, Mar. 2010. 
[30] D. Astély, E. Dahlman, A. Furuskar, Y. Jading, M. Lindstrom and S. Parkvall, "LTE: The evolution of mobile broadband," IEEE Communications Magazine, vol. 47(4), Apr. 2009, pp. 44-51.

[31] R4-070572, "Proposal for LTE channel models,"3GPP, Ericsson, Nokia, Motorola, Rohde \& Schwarz, RAN4-43, May 2007.

[32] ITU-R M.1225 International Telecommunication Union, "Guidelines for evaluation of radio transmission technologies for IMT-2000," 1997.

[33] 3GPP TS 05.05, Radio transmission and reception, Std.

[34] T. Sorensen, P. Mogensen and F. Frederiksen, "Extension of the ITU channel models for wideband (OFDM) systems," in Proc. IEEE VTC'05, vol. 1, Sep. 2005, pp. 392-396.

[35] 3GPP TS 36.101, User equipment (UE) radio transmission and reception, Std.

[36] 3GPP TS 36.104, Base station (BS) radio transmission and reception, Std.

[37] S.A. AL-Semari and T.E. Fuja, "I-Q TCM: Reliable communication over the Rayleigh fading channel close to the cutoff rate," IEEE Transaction on Information Theory, vol. 43(1), Jan.1997, pp. 250-262.

\section{BIOGRAPHIES OF AUTHORS}

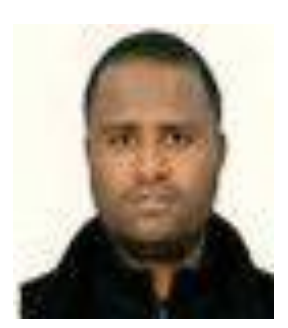

ElarbiAbderraouf was born in Adrar, Algeria. He received the Dipl.El.-Ing. Degree from the University of Molay-Taher-Saida, Algeria in 2008, and his Master from the University of Bechar, Algeria in 2013. Actually, he is a Doctorate student at University of Bechar, Algeria. His main interests are turbo encoding schemes, iterative decoding over fading channels, Correspondance address: Information Processing and Telecommunication Laboratory (LTIT), Tahri Mohammed University, Bechar 08000, Algeria.

E-mail: elarbiabderraouf12@gmail.com

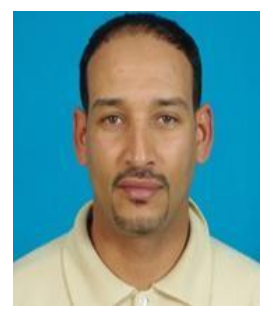

AbdesselamBassou was born in Bechar, Algeria. He received the Dipl.El.-Ing. Degree from the University of Tlemcen, Algeria in 1997, his Master from the University of SidiBel Abbes, Algeria in 2000, and his Doctoral degree Es Science from the University of SidiBel Abbes, Algeria in 2006. Actually, $\mathrm{He}$ is an Associate Professor at University of Bechar, Algeria. His main interests are digital signal processing, turbo encoding schemes and iterative decoding over fading channels, and channel equalization Correspondanceaddress: Information Processing and Telecommunication Laboratory (LTIT), Tahri Mohammed University, Bechar 08000, Algeria. Email: a.bassou@gmail.com

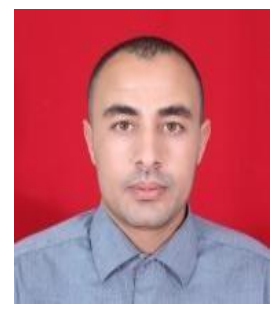

Lahcene Mohamed Rida was born in Adrar, Algeria. He received the Dipl.El.-Ing. Degree from the University of Bechar, Algeria in 2009, and his Master from the University of Bechar, Algeria in 2012, and his Doctoral degree Es Science from the Universityof Bechar, Algeria in 2018 Algeria. His main interests are turbo encoding schemes, iterative decoding over fading channels, and complexity of encoder.Correspondance address: Information Processing and Telecommunication Laboratory (LTIT), Tahri Mohammed University, Bechar 08000, Algeria. E-mail: lahcenereda1@gmail.com 